\title{
DEFORMATION OF DOMAIN AND THE \\ LIMIT OF THE VARIATIONAL EIGENVALUES \\ OF SEMILINEAR ELLIPTIC OPERATORS
}

TETSUTARO SHIBATA

Department of Mathematics

Aichi Prefectural University

3-28, Takada-cho, Mizuho-ku

Nagoya 467, JAPAN

(Received April 11, 1994 and in revised form August 30, 1994)

ABSTRACT. We consider the semilinear elliptic eigenvalue problem

$$
\left\{\begin{array}{rll}
L u+f(x, u)=\mu u & \text { in } & \Omega_{r}(r \geq 0), \\
u=0 & \text { on } & \partial \Omega_{r}
\end{array}\right.
$$

The asymptotic behavior of the variational eigenvalues $\mu=\mu_{n}(r, \alpha)$ obtained by Ljusternik-Schnirelman theory is studied when the domain $\Omega_{0}$ is deformed continuously. We also consider the cases that $\operatorname{Vol}\left(\Omega_{r}\right) \rightarrow 0, \infty$ as $r \rightarrow \infty$.

KEY WORDS AND PHRASES. Deformation of domain, variational eigenvalues.

1991 AMS SUBJECT CLASSIFICATION CODE. 35J60, 35P05, 35P30.

\section{INTRODUCTION.}

In this paper we consider the following semilinear elliptic eigenvalue problem:

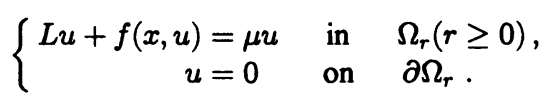

where $\Omega_{r}(r \geq 0)$ is a bounded domain in $R^{N}(N \geq 3)$ with smooth boundary $\partial \Omega_{r}$. Let us assume that

(A.1)

$$
\begin{gathered}
L u:=-\sum_{i, j=1}^{N} \frac{\partial}{\partial x_{j}}\left(a_{i j}(x) \frac{\partial u}{\partial x_{i}}\right)+a_{0}(x) u, \\
a_{0} \geq 0, \quad a_{i j}=a_{j i}(i, j=1,2, \ldots, N),
\end{gathered}
$$

is a formally self-adjoint uniformly elliptic operator with suitably regular, bounded coefficients $a_{i j}, a_{0}$ defined on an open set $\Omega:=\cup_{0 \leq r \leq \infty} \Omega_{r}$. Furthermore, let us assume the following condition (A.2):

(A.2) Suppose that at least one of the following conditions is satisfied:

(A.2.1) $f: \Omega \times R \rightarrow R$ is continuous, odd in $u$, that is, $f(x,-u)=-f(x, u)$, uniformly continuous as a function of $x$ for any fixed $u \in R$ and there exist constants $C_{1}, C_{2} \geq 0$ and $0 \leq h<\frac{N+2}{N-2}$ such that

$$
|f(x, u)| \leq C_{1}|u|^{h}+C_{2} .
$$

Furthermore, for all $(x, u) \in \Omega \times R$, 


$$
F(x, u):=\int_{0}^{u} f(x, t) d t \geq 0 .
$$

(A.2.2) $f$ satisfies (1.2) for $0 \leq h<1+\frac{4}{N}$.

The main results in this paper concern the asymptotic behavior of the variational eigenvalues $\mu=\mu_{n}(r, \alpha)$ of (1.1) obtained by Ljusternik-Schnirelman (LS) theory when the original domain $\Omega_{0}$ is deformed continuously. It is well known (cf. Courant-Hilbert [5]) that the $n$ - th eigenvalues of the linear eigenvalue problems vary continuously when the original domain $\Omega_{0}$ is deformed continuously. However, it seems few results corresponding to this classical linear result are obtained for nonlinear eigenvalue problems. As is well known, the classical results of linear eigenvalues due to CourantHilbert [5] can be partially generalized to the nonlinear case by virtue of (LS)-theory. In particular, Chiappinelli [2-4] considered such problem as (1.1) by using (LS)-theory from a standpoint of $L^{2}$-theory $\left(\mathrm{L}^{2}-(\mathrm{LS})\right.$ theory) and succeeded to obtain the asymptotic formula of variational eigenvalues analogous to Weyl's formula. Hence, it seems available to deal with the problem (1.1) under the framework of $L^{2}$-(LS) theory.

Motivated by this, we shall consider the classical problem of deformation of a domain for semilinear elliptic eigenvalue problem (1.1) by using $L^{2}$-(LS) theory and shall study the asymptotic properties of the variational eigenvalues of (1.1). We also deal with the cases that the volume of $\Omega_{r} \rightarrow 0, \infty$ as $r \rightarrow \infty$.

We shall explain notations before stating our results. Let

$$
x=\left(x_{1}, x_{2}, \ldots, x_{N}\right), D_{\imath}=\frac{\partial}{\partial x_{i}},\left|\Omega_{r}\right|: \text { Lebesgue measure of } \Omega_{r} .
$$

$C$ denotes various constants independent of $r$. For $0 \leq r \leq \infty$, let $X_{r}:=W_{0}^{1,2}\left(\Omega_{r}\right)$ be the usual Sobolev space and $X_{r}^{\prime}$ the dual space of $X_{r}$. Let

$$
\begin{gathered}
(u, v)_{X_{r}}:=\int_{\Omega_{r}} \nabla u \nabla v d x,\|u\|_{X_{r}}^{2}:=(u, u)_{X_{r}}, u, v \in X_{r}, \\
\|u\|_{q, r}:=\left(\int_{\Omega_{r}}|u(x)|^{q} d x\right)^{1 / q}, \\
\langle w, u\rangle_{r}: \text { the pairing between } w \in X_{r}^{\prime} \text { and } u \in X_{r}, \\
\psi_{r, 0}(u):=\frac{1}{2} \sum_{i, j=1}^{N} \int_{\Omega_{r}} a_{i j}(x) D_{i} u D_{j} u d x+\frac{1}{2} \int_{\Omega_{r}} a_{0}(x) u^{2} d x \text { for } u \in X_{r}, \\
\psi_{r}(u)=\psi_{r, 0}(u)+\int_{\Omega_{r}} F(x, u) d x \text { for } u \in X_{r} .
\end{gathered}
$$

For a fixed $\alpha>0$, we set

$$
M_{\alpha, r}:=\left\{u \in X_{r}:\|u\|_{2, r}^{2}=\alpha^{2}\right\} .
$$

For a closed, symmetric $(-u \in K$ whenever $u \in K)$ subset $K \subset X_{r}$ with $0 \notin K$, the genus of $K$ is defined by

$$
\gamma(K)=\min \left\{n \in N: \text { there exists } H: K \rightarrow R^{n} \backslash\{0\}, \text { continuous and odd }\right\} .
$$

We put

$$
K_{n, r}(\alpha)=\left\{K \subset M_{\alpha, r}: \quad \text { compact, symmetric, } 0 \notin K, \gamma(K)=n\right\}
$$


We denote by $\mu_{n}(r)$ the $n-t h$ eigenvalue of the corresponding linear eigenvalue problem (i e., $f \equiv 0$ ) of (1.1).

Now we shall give the definition of the variational eigenvalue $\mu_{n}(r, \alpha)$. We call $\mu_{n}(r, \alpha)$ the $n-t h$ variational eigenvalue of $\left(\begin{array}{ll}1 & 1)\end{array}\right)$ if the associated eigenfunction $u_{n}(r, \alpha) \in M_{\alpha, r}$ satisfies

$$
2 \psi_{r}\left(u_{n}(r, \alpha)\right)=C_{n}(r, \alpha):=\inf _{K \in K_{n, r}} \sup _{u \in K} 2 \psi_{r}(u)
$$

It should be remarked that $\mu_{n}(r, \alpha)$ is given explicitly by

$$
\mu_{n}(r, \alpha)=\frac{\left\langle\psi_{r}^{\prime}\left(u_{n}(r, \alpha)\right), u_{n}(r, \alpha)\right\rangle_{r}}{\alpha^{2}}
$$

where $\psi_{r}^{\prime}$ is the Gâteaux derivative of $\psi_{r}$.

We can now state our assumptions and present our main results. We assume that $\Omega_{r}$ satisfies one of the following conditions (D.1)-(D.3):

(D.1) There exists a bounded domain $\Omega_{\infty} \subset R^{N}$ with smooth boundary and $C^{1}$-diffeomorphism $\Phi_{r}: \Omega_{r} \rightarrow \Omega_{\infty}$ such that as $r \rightarrow \infty$

$$
J_{r}(x):=J_{\Phi_{r}}(x) \rightarrow I_{N} \text { uniformly in } x \in \Omega_{r},
$$

where $J_{\Phi_{r}}(x)$ and $I_{N}$ stand for the Jacobian matrix of $\Phi_{r}$ and the unit $N \times N$ matrix, respectively.

(D.2)

$$
\lim _{r \rightarrow \infty}\left|\Omega_{r}\right|=0, \lim _{r \rightarrow \infty} \mu_{n}(r)=\infty .
$$

(D.3) There exists a $C^{1}$-diffeomorphism $\Phi_{r}: \Omega_{0} \rightarrow \Omega_{r}$, a constant $C>0$ and a positive function $\lambda(r)(\lambda(r) \rightarrow \infty$ as $r \rightarrow \infty)$ such that for any $r \geq 0$ and $x \in \Omega_{0}$

$$
C^{-1} \lambda(r) \leq \lambda_{1, r}(x) \leq \lambda_{2, r}(x) \leq \ldots \leq \lambda_{N, r}(x) \leq C \lambda(r) .
$$

where $\lambda_{\jmath, r}(x)(j=1,2, \ldots, N)$ is the $j$-th eigenvalue of the $N \times N$ symmetric matrix $J_{r}(x)^{t} J_{r}(x)\left(J_{r}(x)^{t}\right.$ : the transposed matrix of $\left.J_{r}(x)\right)$. Furthermore, there exist constants $k$ with $0 \leq k \leq h$ and $C>0$ such that for any $u \geq 0$ and $x \in \Omega$

$$
C u^{k} \leq|f(x, u)| \text {. }
$$

THEOREM 1. Assume (A.1), (A.2) and (D.1). Let $\left\{\mu_{n}(\infty, \alpha)\right\}$ be the set of the $n-t h$ variational eigenvalues of (1.1) with $\Omega_{r}$ replaced by $\Omega_{\infty}$. Then for any fixed $\alpha>0$ and $n \in N$, there exists $\mu_{\infty} \in\left\{\mu_{n}(\infty, \alpha)\right\}$ and a sequence $\left\{\mu_{n}\left(r_{j}, \alpha\right)\right\}\left(r_{j} \rightarrow \infty\right.$ as $\left.j \rightarrow \infty\right)$ such that

$$
\mu_{\infty}=\lim _{j \rightarrow \infty} \mu_{n}\left(r_{\jmath}, \alpha\right) \text {. }
$$

THEOREM 2. Suppose that one of the following conditions is satisfied:

(1) (A.1), (A.2.2), (1.3) and (D.2),

(2) (A.1), (A.2.1), (D.2) and the following (1.7): for all $(x, u) \in \Omega \times R$

$$
f(x, u) u-2 F(x, u) \geq 0 .
$$

Then for any fixed $\alpha>0, \mu_{n}(r, \alpha) \rightarrow \infty$ as $r \rightarrow \infty$.

THEOREM 3. Assume (A.1), (A.2.1) and (D.3). Then for any fixed $\alpha>0$, the following properties hold:

(a) If $k>1$ and $C_{2}=0$, then $\mu_{n}(r, \alpha) \rightarrow 0$ as $r \rightarrow \infty$.

(b) If $N>4,0 \leq h<1,0 \leq k<\frac{\sqrt{N}-2}{\sqrt{N}+2}$ and $f(x, u)>0$ for $x \in \Omega$ and $u>0$, then $\mu_{n}(r, \alpha) \rightarrow \infty$ as $r \rightarrow \infty$. 
REMARKS. (1) The restriction of $h$ in (A 2 2) comes from the existence result of variational eigenvalues For the existence of variational eigenvalues on the manifold $M_{\alpha, r}$, we need to show that $\psi_{r}$ is bounded below on $M_{\alpha, r}$ Clearly, $\psi_{r}$ is bounded below if (13) is satisfied. Hence, the existence theorem holds for $0 \leq h<\frac{N+2}{N-2}$ under the condition (A 2 1) (see [2, Lemma 2])

If $\left(\begin{array}{ll}13 \\ 3\end{array}\right)$ is not assumed, then in order to obtain the boundedness from below, we apply the following interpolation inequality, which is a direct consequence of the Sobolev embedding theorem for $u \in W_{0}^{1,2}(\Omega)$

$$
\|u\|_{h+1}^{h+1} \leq C\|\nabla u\|_{2}^{h+1-\gamma}\|u\|_{2}^{\gamma}
$$

where $\gamma: 0 \leq \gamma \leq \beta(h):=\left(N / p_{0}\right)\left(p_{0}-(h+1)\right), p_{0}=\frac{2 N}{N-2}$. Then the inequality (1.8) and direct calculation lead us to the following inequality (see [4, Theorem 1]): on $M_{\alpha, r}$

$$
\psi_{r}(u) \geq C\|u\|_{X_{r}}^{2}-C \alpha^{\beta}\|u\|_{X_{r}}^{N(h-1) / 2}-C \alpha^{2}-C \alpha .
$$

Here $\beta=(p+1)-(p-1) N / 2$. Hence, the restriction $h<1+\frac{4}{N}$ occurs.

(2) The condition (D.2) is satisfied, for example, under the condition that the lattice packing density $\delta\left(\Delta_{\mathrm{r}}\right)$ is bounded below (see, e.g. Urakawa [6]).

(3) Under the suitable regularity condition on $a_{\imath \jmath}, a_{0}$ and $f,(1.1)$ is equivalent to its weak formulation, namely, that of finding $u \in X_{r}$ and $\mu \in R$ such that for any $v \in X_{r}$

$$
\sum_{\imath, \jmath=1}^{N} \int_{\Omega_{r}} a_{\imath \jmath}(x) D_{\imath} u D_{\jmath} v d x+\int_{\Omega r} a_{0}(x) u v d x+\int_{\Omega_{r}} f(x, u) v d x=u \int_{\Omega_{r}} u v d x .
$$

Hence, in what follows, we consider (1.9) instead of (1.1)

\section{PROOF OF THEOREM 1}

We begin with showing the following important fact.

LEMMA 2.1. Assume (A.1), (A.2) and (D.1). Then for any fixed $\alpha>0$ and $n \in N$,

$$
\lim _{r \rightarrow \infty} C_{n}(r, \alpha)=C_{n}(\infty, \alpha)
$$

PROOF. Supposing that the assertion does not hold, we shall derive a contraction. Since $C_{n}(r, \alpha)$ does not converge to $C_{n}(\infty, \alpha)$ as $r \rightarrow \infty$, we can choose a constant $\delta>0$ and a sequence $\left\{r_{\jmath}\right\}_{j=1}^{\infty}$ such that $r_{\jmath} \rightarrow \infty$ as $j \rightarrow \infty$ and

$$
\left|C_{n}\left(r_{\jmath}, \alpha\right)-C_{n}(\infty, \alpha)\right| \geq \delta .
$$

In what follows, we denote any subsequence of $\left\{r_{\jmath}\right\}$ by $\left\{r_{\jmath}\right\}$ again. There are two possible cases: there exists a subsequence of $\left\{r_{j}\right\}$ such that

(1) $C_{n}\left(r_{\jmath}, \alpha\right) \geq C_{n}(\infty, \alpha)$,

(2) $C_{n}\left(r_{\jmath}, \alpha\right) \leq C_{n}(\infty, \alpha)$.

Since both cases can be treated similarly, we consider only the case that (1) holds. By definition of $C_{n}(\infty, \alpha)$, there exists $K \in K_{n, \infty}(\alpha)$ such that

$$
0 \leq \sup _{u \in K} 2 \psi_{\infty}(u)-C_{n}(\infty, \alpha)<\frac{\delta}{2}
$$

We put

$$
K_{\jmath}=\left\{\mu \in X_{r}: u=v \circ \Phi_{r_{\jmath}}, v \in K\right\}, K_{j, 1}=\left\{v=\frac{\alpha u}{\|u\|_{2, r_{\jmath}}}: u \in K_{\jmath}\right\} \subset M_{\alpha, r}
$$


Then it is clear that the mapping $G_{1}: K \rightarrow K_{\mathrm{J}}$ and $G_{2}: K_{\mathrm{J}} \rightarrow K_{\jmath, 1}$ are odd homeomorphisms under the condition (D 1) More precisely,

$$
G_{1}(-v)=-v \circ \Phi_{r_{j}}=-G_{1}(v), \quad v \in K, G_{2}(-u)=\frac{-\alpha u}{\|u\|_{2, r},}=-G_{2}(u), \quad u \in K_{\jmath},
$$

and by (D.1) $G_{1}$ and $G_{2}$ are homeomorphisms. Since the genus is preserved by odd homeomorphisms, we have

$$
\gamma\left(K_{\jmath, 1}\right)=\gamma\left(K_{\jmath}\right)=\gamma(K)=n
$$

and hence $K_{\jmath, 1} \in K_{n, r_{j}}(\alpha)$. Then, noting that $C_{n}\left(r_{\jmath}, \alpha\right) \leq \sup _{u \in K_{j, 1}} 2 \psi_{r_{\jmath}}(u)$, we obtain by (2.2) that

$$
\begin{aligned}
C_{n}\left(r_{\jmath}, \alpha\right)- & C_{n}(\infty, \alpha)=C_{n}\left(r_{\jmath}, \alpha\right)-\sup _{u \in K_{j, 1}} 2 \psi_{r_{j}}(u)+\sup _{u \in K_{\jmath, 1}} 2 \psi_{r_{j}}(u) \\
& +\sup _{u \in K_{j}} 2 \psi_{r_{j}}(u)-\sup _{u \in K_{\jmath}} 2 \psi_{r_{j}}(u)-\sup _{u \in K} 2 \psi_{\infty}(u) \\
& +\sup _{u \in K} 2 \psi_{\infty}(u)-C_{n}(\infty, \alpha) \\
& \leq\left|\sup _{u \in K_{\jmath, 1}} 2 \psi_{r_{j}}(u)-\sup _{u \in K_{\jmath}} 2 \psi_{r_{j}}(u)\right|+\left|\sup _{u \in K_{\jmath}} 2 \psi_{r_{\jmath}}(u)-\sup _{u \in K} 2 \psi_{\infty}(u)\right|+\frac{\delta}{2} \\
& :=I_{1}+I_{2}+\frac{\delta}{2} .
\end{aligned}
$$

We shall estimate $I_{1}$. At first, we consider the case that there exists a subsequence of $\left\{r_{j}\right\}$ such that

$$
\sup _{u \in K_{\jmath}} 2 \psi_{r,}(u) \geq \sup _{u \in K_{j, 1}} 2 \psi_{r,}(u)
$$

Since $K_{j}$ is a compact in $X_{r}$, we can choose $v_{j} \in K_{j}$ which attains the supremum of the left hand side of (2.4). We put $\alpha_{\jmath}=\left\|v_{\jmath}\right\|_{2, r_{\jmath}}$ and $w_{\jmath}=\frac{\alpha v_{\jmath}}{\alpha_{\jmath}} \in K_{\jmath, 1}$. Then by (1.5), we easily obtain that $\alpha_{\jmath} \rightarrow \alpha$ as $j \rightarrow \infty$, and moreover,

$$
\sup _{j \in J, u \in K_{j}}\|u\|_{X_{r}} \leq C<\infty
$$

from which it follows that

$$
\left\|v_{j}-w_{j}\right\|_{X_{r_{j}}} \leq C\left(1-\frac{\alpha}{\alpha_{j}}\right)
$$

Then using (1.5), (2.5) and (2.6), we obtain by simple calculation that $j \rightarrow \infty$

$$
I_{1} \leq 2 \psi_{r_{j}}\left(v_{j}\right)-2 \psi_{r_{j}}\left(w_{j}\right) \leq C\left(1-\frac{\alpha}{\alpha_{j}}\right) \rightarrow 0
$$

In the same way as that used above, we can also choose a subsequence of $\left\{r_{j}\right\}$ such that $I_{1} \rightarrow 0$ as $j \rightarrow \infty$ in the case that there exists infinitely many $j$ satisfying

$$
\sup _{u \in K_{j}} 2 \psi_{r_{j}}(u)<\sup _{u \in K_{j, 1}} 2 \psi_{r_{j}}(u)
$$

Consequently, we can choose a subsequence of $\left\{r_{j}\right\}$ such that $I_{1} \rightarrow 0$ as $j \rightarrow \infty$.

As for $I_{2}$, by the same method as that used above, we can also choose a subsequence of $\left\{r_{j}\right\}$ such that $I_{2} \rightarrow 0$ as $j \rightarrow \infty$. Thus, it follows from (2.3) that $C_{n}\left(r_{\jmath}, \alpha\right)-C_{n}(\infty, \alpha)<\frac{3 \delta}{4}$ for sufficiently large $j$. This contradicts (2.1). Thus the proof is complete. 
For $r_{0} \gg 1$, we consider the mapping $\Phi_{r_{0}}^{-1} \circ \Phi_{r}: \Omega_{r_{0}}$. Since by (1.5), $J_{\Phi_{r}^{-1} \circ \Phi_{r}}(x) \rightarrow I_{N}$ uniformly in $x \in \Omega_{r}$ as $r \rightarrow r_{0}$, we obtain the following corollary by the same argument as that used in Lemma 2.1.

COROLLARY 2.2 Assume (A.1), (A.2) and (D.1). Then for a fixed $\alpha>0$ and $n \in N, C_{n}(r, \alpha)$ is continuous with respect to $r$ for $1 \ll r \leq \infty$.

Let us introduce the following lemma due to Chiappinelli [2].

LEMMA 2.3 ([2, LEMMA 5]). If (A.1), (A.2 1) and either (D 1) or (D 2) are satisfied, then there exist constants $C_{3}, C_{4}>0$ such that for $0 \leq r \leq \infty$ and $n \in N$

$$
\left|C_{n}(r, \alpha)-\alpha^{2} \mu_{n}(r, \alpha)\right| \leq C_{3} \alpha^{\beta}\left(C_{n}(r, \alpha)\right)^{N(h-1) / 4}+C_{4} \alpha,
$$

where $\beta=(p+1)-(p-1) N / 2$

LEMMA 2.4. Assume (A 1), (A.2) and (D.1). Then $\left\{\mu_{n}(r, \alpha): r \gg 1\right\}$ is bounded.

PROOF. If (A.1), (A.2.1) and (D.1) are satisfied, then the assertion is the immediate consequence of Corollary 22 and (2.7).

Next, assume that (A.1), (A.2.2) and (D.1) are satisfied. At first, let $h>1$. Then

$$
\begin{aligned}
\left|C_{n}(r, \alpha)-\alpha^{2} \mu_{n}(r, \alpha)\right| & =\mid \psi_{r, 0}\left(u_{n}(r, \alpha)\right)+2 \int_{\Omega_{r}} F\left(x, u_{n}(r, \alpha)\right) d x \\
& -\left(\psi_{r, 0}\left(u_{n}(r, \alpha)\right)+\int_{\Omega_{r}} f\left(x, u_{n}(r, \alpha)\right) u_{n}(r, \alpha) d x\right) \mid \\
& =\left|2 \int_{\Omega_{r}} F\left(x, u_{n}(r, \alpha)\right) d x-\int_{\Omega_{r}} f\left(x, u_{n}(r, \alpha)\right) u_{n}(r, \alpha) d x\right| \\
& \leq C \int_{\Omega_{r}}\left|u_{n}(r, \alpha)\right|^{h+1} d x+C_{2} \alpha .
\end{aligned}
$$

By setting $\gamma=\beta:=b(h)=(h+1)-(h-1) N / 2$ in (1.8), we obtain that for $u \in X_{r}$

$$
\int_{\Omega_{r}}|u|^{h+1} d x \leq C \alpha^{\beta}\left(\int_{\Omega_{r}}|\nabla u|^{2} d x\right)^{N(h-1) / 4} .
$$

Then we obtain by (2.8) and (2.9) that

$$
\left|C_{n}(r, \alpha)-\alpha^{2} \mu_{n}(r, \alpha)\right| \leq C \alpha^{\beta}\left(\int_{\Omega_{r}}\left|\nabla u_{n}(r, \alpha)\right|^{2} d x\right)^{N(h-1) / 4}+C \alpha .
$$

By ellipticity, we have by (2.8) and (2.9) that

$$
\begin{aligned}
\int_{\Omega_{r}}\left|\nabla u_{n}(r, \alpha)\right|^{2} d x & \leq C \psi_{r, 0}\left(u_{n}(r, \alpha)\right)=C\left|C_{n}(r, \alpha)-2 \int_{\Omega_{r}} F\left(x, u_{n}(r, \alpha)\right) d x\right| \\
& \leq C\left(C_{n}(r, \alpha)+\int_{\Omega_{r}}\left|u_{n}(r, \alpha)\right|^{h+1} d x\right) \\
& \leq C\left(C_{n}(r, \alpha)+C \alpha^{\beta}\left(\int_{\Omega_{r}}\left|\nabla u_{n}(r, \alpha)\right|^{2} d x\right)^{N(h-1) / 4}+C \alpha\right)
\end{aligned}
$$

Since $1<h<1+\frac{4}{N}$ and $C_{n}(r, \alpha)$ is bounded by Corollary 2.2 , it is obvious by (2.11) that

$$
\left\|u_{n}(r, \alpha)\right\|_{X_{r}} \leq C \text {. }
$$

Now, by (2.10) and (2.12) we obtain

$$
\left|\alpha^{2} \mu_{n}(r, \alpha)\right| \leq\left|C_{n}(r, \alpha)\right|+\left|C_{n}(r, \alpha)-\alpha^{2} \mu_{n}(r, \alpha)\right| \leq C+C \alpha .
$$

Thus the proof is complete for $h>1$. 
If $0 \leq h \leq 1$, then by Chiappinelli [3, Theorem 2.2] we have

$$
\left|\mu_{n}(r, \alpha)-\mu_{n}(r)\right| \leq C .
$$

Thus the conclusion immediately follows from (2 14)

By Lemma 24 , we may assume that $\mu_{\infty}=\lim _{r \rightarrow \infty} \mu_{n}(r, \alpha)$; otherwise, we choose a suitable subsequence. Let

$$
v_{n}(r)=u_{n}(r, \alpha) \circ \Phi_{r}^{-1} \in X_{\infty}, \alpha_{r}=\left\|v_{n}(r)\right\|_{2, \infty}, w_{n}(r)=\frac{\alpha v_{n}(r)}{\alpha_{r}} \in M_{\alpha, \infty} .
$$

LEMMA 2.5. Assume (A.1), (A 2) and (D.1). Then

$$
\sup _{r \gg 1}\left\|w_{n}(r)\right\|_{X_{\infty}} \leq C<\infty .
$$

PROOF. At first, we assume (A.1), (A.2.1) and (D.1). Then by (A.1) and (1.3) there exists a constant $C_{6} \geq 0$ such that

$$
\left\|u_{n}(r, \alpha)\right\|_{X_{r}}^{2} \leq C_{6} \psi_{r, 0}\left(u_{n}\right) \leq C_{6} \psi_{r}\left(u_{n}\right)=C_{6} C_{n}(r, \alpha) .
$$

Then by Corollary 2.2 it is clear that $C_{n}(r, \alpha)$ is bounded for $1 \ll r \leq \infty$. Therefore, we obtain our assertion by (1.5) and (2.16).

Next, we assume that (A.1), (A.2.2) and (D.1). Multiplying (1.1) by $\mu_{n}(r, \alpha)$ and integration by parts together with the fact that $u_{n}(r, \alpha) \in M_{\alpha, r}$, we obtain

$$
2 \psi_{r, 0}\left(u_{n}(r, \alpha)\right)+\int_{\Omega_{r}} f\left(x, u_{n}(r, \alpha)\right) u_{n}(r, \alpha) d x=\mu_{n}(r, \alpha) \alpha^{2} .
$$

If $0 \leq h \leq 1$, then by (1.2) and Hölder's inequality we obtain

$$
\begin{aligned}
\left|\int_{\Omega_{r}} f\left(x, u_{n}(r, \alpha)\right) u_{n}(r, \alpha) d x\right| & \leq \int_{\Omega_{r}} C_{1} \mid\left(\left.u_{n}(r, \alpha)\right|^{h+1}+C_{2}\left|u_{n}(r, \alpha)\right|\right) d x \\
& \leq C_{1} \alpha^{h+1}\left|\Omega_{r}\right|^{(1-h) / 2}+C_{2} \alpha\left|\Omega_{r}\right| .
\end{aligned}
$$

Since $\left\{\mu_{n}(r, \alpha): r \gg 1\right\}$ is bounded by Lemma 2.4 , we obtain by (2.17) and (2.18) that

$$
\left\|u_{n}(r, \alpha)\right\|_{X_{r}}^{2} \leq C \psi_{r, 0}\left(u_{n}(r, \alpha)\right)<C .
$$

If $h>1$, then our conclusion is exactly (2.12). Now (2.15) is an immediate consequence of (1.5).

LEMMA 2.6. There exists a subsequence of $\left\{w_{n}(r)\right\}$ such that as $r \rightarrow \infty$

$$
\psi_{\alpha, \infty}^{\prime}\left(w_{n}(r)\right):=\psi_{\infty}^{\prime}\left(w_{n}(r)\right)-\frac{\left\langle\psi_{\infty}^{\prime}\left(w_{n}(r)\right), w_{n}(r)\right\rangle_{r}}{\alpha^{2}} w_{n}(r) \rightarrow 0 \text { in } X_{\infty}^{\prime}
$$

PROOF. We set $S_{\infty}:=\left\{v \in X_{\infty}:\|v\|_{X_{\infty}}=1\right\}$. For $v \in S_{\infty}$, we put $v_{r}=v \circ \Phi_{r} \in X_{r}$. For $p=\left(p_{1}, p_{2}, \ldots, p_{N}\right) \in R^{N}$ and $q=\left(q_{1}, q_{2}, \ldots, q_{N}\right) \in R^{N}$, we set $(p, q)_{i, j}=p_{\imath} q_{j}$ for $i, j=1,2, \ldots, N$. Then we obtain that 


$$
\begin{aligned}
& \left\|\psi_{\alpha, \infty}^{\prime}\left(w_{r}(r)\right)\right\|_{\lambda_{\infty}^{\prime}}=\sup _{v \in S_{(x,}}\left\langle\psi_{\alpha, \infty}\left(w_{n}(r)\right), v\right\rangle_{\infty} \\
& =\sup _{v \in S_{\infty}}\left\{\int_{\Omega_{\infty}} \sum_{\imath, J=1}^{N} a_{\imath \jmath}(y) D_{\imath} w_{n}(r) D_{\jmath} v d y+\int_{\Omega_{\infty}} f\left(y, w_{n}(r)\right) v d y\right. \\
& \left.-\frac{1}{\alpha^{2}}\left(2 \psi_{\infty, 0}\left(w_{n}(r)\right)+\int_{\Omega_{\infty}} f\left(y, w_{n}(r)\right) w_{n}(r) d y\right) \int_{\Omega_{\infty}} w_{n}(r) v d y\right\} \\
& =\sup \left\{\frac{\alpha}{\alpha_{r}} \int_{\Omega_{r}} \sum_{i, J=1}^{N} a_{\imath \jmath}\left(\Phi_{r}(x)\right)\left(J_{r}(x) \nabla u_{n}(r, \alpha), J_{r}(x) \nabla v_{r}\right)_{\imath \jmath}\left|J_{r}(x)\right| d x\right. \\
& +\int_{\Omega_{r}} f\left(\Phi_{r}(x), \frac{\alpha u_{n}(r, \alpha)}{\alpha_{r}}\right) v_{r}\left|J_{r}(x)\right| d x \\
& -\frac{1}{\alpha \alpha_{r}}\left(\frac{\alpha^{2}}{\alpha_{r}^{2}} \int_{\Omega_{r}} \sum_{i, j=1}^{N} a_{\imath \jmath}\left(\Phi_{r}(x)\right)\left(J_{r}(x) \nabla u_{n}(r, \alpha), J_{r}(x) \nabla u_{n}(r, \alpha)\right)_{\imath, j}\left|J_{r}(x)\right| d x\right. \\
& \left.\left.+\frac{\alpha}{\alpha_{r}} \int_{\Omega_{r}} f\left(\Phi_{r}(x), \frac{\alpha u_{n}(r, \alpha)}{\alpha_{r}}\right) u_{n}(r, \alpha)\left|J_{r}(x)\right| d x\right) \int_{\Omega_{r}} u_{n}(r, \alpha) v_{r}\left|J_{r}(x)\right| d x\right\}
\end{aligned}
$$

On the other hand, since $u_{n}(r, \alpha)$ satisfies $\left(\begin{array}{ll}1 & 1\end{array}\right)$, we have for any $v_{r}$

$$
\begin{aligned}
& \int_{\Omega_{r}} \sum_{i, j=1}^{N} a_{\imath \jmath}(x) \nabla u_{n}(r, \alpha) \nabla v_{r} d x+\int_{\Omega_{r}} f\left(x, u_{n}(r, \alpha)\right) v_{r} d x \\
& =\alpha^{-2}\left(2 \psi_{r, 0}\left(u_{n}(r, \alpha)\right)+\int_{\Omega_{r}} f\left(x, u_{n}(r, \alpha)\right) u_{n}(r, \alpha) d x\right) \int_{\Omega_{r}} u_{n}(r, \alpha) v_{r} d x .
\end{aligned}
$$

We compare (2.21) with (2.22). Then noting that $\sup _{r \gg 1, v \in S_{\infty}}\left\|v_{r}\right\|_{X_{r}}<C$ and $\alpha_{r} \rightarrow \alpha$ as $r \rightarrow \infty$, we can easily obtain (2.20) by direct calculation using (1.5), (2.21) and (2.22).

Now we are in the position to prove Theorem 1.

PROOF OF THEOREM 1. Since we know from Chiappinelli [2, Lemma 1], [4, Theorem 1] that the functional $\psi_{\infty}$ satisfies the parais-Smale condition on $M_{\alpha, \infty}$, that is, the sequence $\left\{y_{\xi}\right\} \subset M_{\alpha, \infty}$ satisfying (2.15) and (2.20) contains a strongly convergent subsequence in $X_{\infty}$. Hence, by Lemma 2.5 and Lemma 2.6 we can choose a subsequence of $\left\{w_{r}(r)\right\}$, which converges strongly to some $u_{\infty} \in M_{\alpha, \infty}$ in $X_{\infty}$. By definition of $w_{n}(r)$, we can easily find by letting $r \rightarrow \infty$ in (1.9) that $u_{\infty}$ and $\mu_{\infty}$ satisfies (1.9).

Finally, we have only to show that $2 \psi_{\infty}\left(u_{\infty}\right)=C_{n}(\infty, \alpha)$, which follows immediately from (1.4), (1.5), the definition of $w_{n}(r)$ and Lemma 2.1. Thus we get Theorem 1.

\section{PROOF OF THEOREM 2 AND THEOREM 3.}

PROOF OF THEOREM 2. If we assume (A.1), (A.2.2), (1.3) and (D.2), then by Chiappinelli [2, Theorem 6] we know that for $1<h<1+\frac{4}{N}$

$$
\mu_{n}(r, \alpha)=\mu_{n}(r)+O\left(\mu_{n}(r)^{N(h-1) / 4}\right)
$$

this implies our consequence. If $0 \leq h \leq 1$, then our conclusion follows immediately from (2.14) and (D.2).

If we assume (A.1), (A.2.1), (D.2) and (1.7), then we obtain by (1.3), (1.7) and (2.17) that 


$$
\begin{aligned}
\alpha^{2} \mu_{n}(r) & =\inf _{K \in K_{n, r}} \sup _{u \in K} 2 \psi_{r, 0}(u) \leq \inf _{K \in K_{n, r}} \sup _{u \in K} 2 \psi_{r}(u) \\
& =C_{n}(r, \alpha)=\psi_{r, 0}\left(u_{n}(r, \alpha)\right)+2 \int_{\Omega_{r}} F\left(x, u_{n}(r, \alpha)\right) d x \\
& \leq \psi_{r, 0}\left(u_{n}(r, \alpha)\right)+\int f\left(x, u_{n}(r, \alpha)\right) u_{n}(r, \alpha) d x=\alpha^{2} \mu_{n}(r, \alpha) .
\end{aligned}
$$

Thus the proof is complete

We shall prove Theorem 3 in the rest of this section.

PROOF OF THEOREM 3 (a). Under the condition (D 3), it is easy to show by mini-max principle that $\mu_{n}(r) \rightarrow 0$ as $r \rightarrow \infty$. Furthermore, it is easy to see that the constants $C_{3}$ and $C_{5}$ in Lemma 2.3 do not depend on $\left|\Omega_{r}\right|$ Since $C_{2}=0$, we know from Chiappinelli [2, Lemma 5] that $C_{4}=0$ in (2.7) and that

$$
C_{n}(r, \alpha) \leq \alpha^{2} \mu_{n}(r)+C_{7} \alpha^{\beta+N(h-1) / 2}\left(\mu_{n}(r)\right)^{N(h-1) / 4},
$$

where $\beta=(h+1)-(h-1) N / 2$. Hence, we see that $C_{n}(r, \alpha) \rightarrow 0$ as $r \rightarrow \infty$. Now Theorem 3(a) follows immediately from Lemma 2.3 .

PROOF OF THEOREM 3(b). We assume that $\left\{\mu_{n}(r, \alpha)\right\}$ is bounded and derive a contradiction. For $u \in M_{\alpha, r}$, we write $v_{r}=u\left(\Phi_{r}(x)\right) \in X_{0}$. By (D.3), we obtain that

$$
\begin{gathered}
\alpha^{2}=\int_{\Omega_{r}}|u(y)|^{2} d y=\int_{\Omega_{0}}\left|v_{r}(x)\right|^{2}\left|J_{r}(x)\right| d x \\
C^{-1} \lambda(r)^{N / 2} \int_{\Omega_{0}}\left|v_{r}(x)\right|^{2} d x \leq \int_{\Omega_{0}}\left|v_{r}(x)\right|^{2}\left|J_{r}(x)\right| d x \leq C \lambda(r)^{N / 2} \int_{\Omega_{0}}\left|v_{r}(x)\right|^{2} d x .
\end{gathered}
$$

We set $v_{n}(r, \alpha)=u_{n}(r, \alpha) \circ \Phi_{r}(x) \in X_{0}, w_{n}(r)=\frac{v_{n}(r)}{\left\|v_{n}(r)\right\|_{2,0}}$. Then it follows from (1.5), (1.6) and (3.1) that

$$
\begin{aligned}
\alpha^{2} \mu_{n}(r, \alpha) & =2 \psi_{r, 0}\left(u_{n}(r, \alpha)\right)+\int_{\Omega_{r}} f\left(x, u_{n}(r, \alpha)\right) u_{n}(r, \alpha) d x \\
& \geq C \int_{\Omega_{r}}\left|u_{n}(r, \alpha)\right|^{k+1} d y=C \int_{\Omega_{0}}\left|v_{n}(r, \alpha)\right|^{k+1}\left|J_{r}(x)\right| d x \\
& \geq C \lambda(r)^{N / 2}\left\|v_{n}(r, \alpha)\right\|_{2}^{k+1} \int_{\Omega_{0}}\left|w_{n}(r, \alpha)\right|^{k+1} d x \\
& \geq C \lambda(r)^{N(1-k) / 4} \int_{\Omega_{0}}\left|w_{n}(r, \alpha)\right|^{k+1} d x
\end{aligned}
$$

Since $\left\{\left\|u_{n}(r, \alpha)\right\|_{X_{r}}\right\}$ is bounded by (3.3), we obtain by (D.3) that

$$
\begin{aligned}
\int_{\Omega_{0}}\left|\nabla v_{n}(r, \alpha)(x)\right|^{2} d x & =\int_{\Omega_{r}}\left|\nabla u_{n}(r, \alpha)(y)\right|^{2}\left|J_{r}^{-1}(y)\right| d y \\
& \leq C \lambda(r)^{N / 2} \int_{\Omega_{r}}\left|\nabla u_{n}(r, \alpha)(y)\right|^{2} d y \leq C \lambda(r)^{N / 2},
\end{aligned}
$$

which together with (3.2) and (3.3) implied that

$$
\int_{\Omega_{0}}\left|w_{n}(r, \alpha)(x)\right|^{2} d x=\left\|v_{n}(r, \alpha)\right\|_{2,0}^{-2} \int_{\Omega_{0}}\left|\nabla v_{n}(r, \alpha)\right|^{2} d x \leq C \lambda(r)^{N}
$$

We obtain by Berger $[1$, p. 43] that

$$
\left\|w_{n}(r, \alpha)\right\|_{2,0} \leq C\left\|w_{n}(r, \alpha)\right\|_{k+1,0}^{\beta}\left\|w_{n}(r, \alpha)\right\|_{X_{0}}^{1-\beta},
$$

where $\beta=\frac{N(1-k)}{N(1-k)+2(1+k)}$. Hence, it follows from (3.3)-(3.5) that 


$$
1 \leq C \lambda(r)^{-\beta N(1-k) /(4(1+k))} \lambda(r)^{N(1-\beta) / 2}=C \lambda(r)^{N / 2-N(3+k) \beta /(4(1+k))} .
$$

Then the exponent of $\lambda(r)$ in (3.6) is: if $N>4$ and $k<\frac{\sqrt{N}-2}{\sqrt{N}+2}$, then

$$
\frac{N}{4}\left(2-\frac{3+k}{1+k} \beta\right)=\frac{N}{4(1+k)} \frac{(4-N) k^{2}+2(N+4) k+(4-N)}{N(1-k)+2(1+k)}<0 .
$$

This is a contradiction, since $\lambda(r) \rightarrow \infty$ as $r \rightarrow \infty$ Thus we get Theorem 3(b).

ACKNOWLEDGMENT. The author would like to express his sincere gratitude to the referees for their helpful comments and suggestions.

\section{REFERENCES}

[1] BERGER, M., Nonlinearity and Functional Analysis, Academic Press, New York, 1977

[2] CHIAPPINELLI, R, On spectral asymptotics and bifurcation for elliptic operators with odd superlinear term, Nonlinear Analysis, 13 (1989), 871-878.

[3] CHIAPPINELLI, R., On the eigenvalues and the spectrum for a class of semilinear operators, Boll. Un. Mat. Ital. B(6), 4 (1985), 867-882.

[4] CHIAPPINELLI, R., Remarks on bifurcation for elliptic operators with odd nonlinearity, Israel J. Math., 65 (1989), 285-292.

[5] COURANT, R. and HILBERT, D., Methods of Mathematical Physics, Vol. I, Intersci. Publ., New York, 1953.

[6] URAKAWA, H., Lower bounds for the eigenvalues of fixed vibration membrane problems, Tôhoku Math. J., 36 (1984), 185-189. 


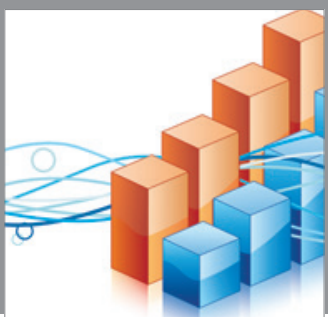

Advances in

Operations Research

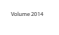

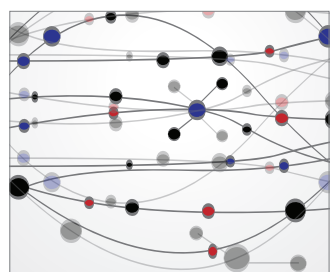

\section{The Scientific} World Journal
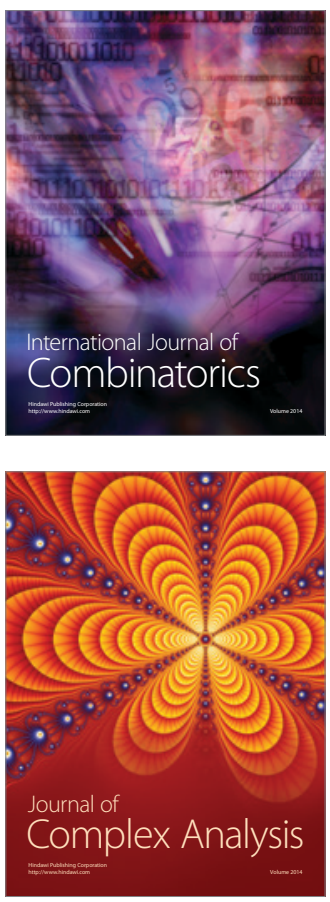

International Journal of

Mathematics and

Mathematical

Sciences
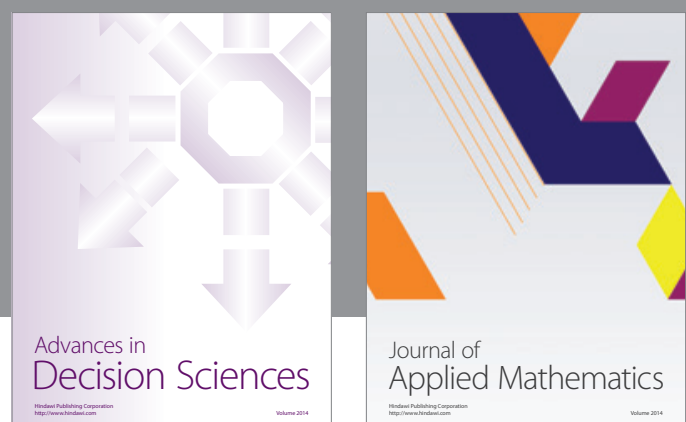

Journal of

Applied Mathematics
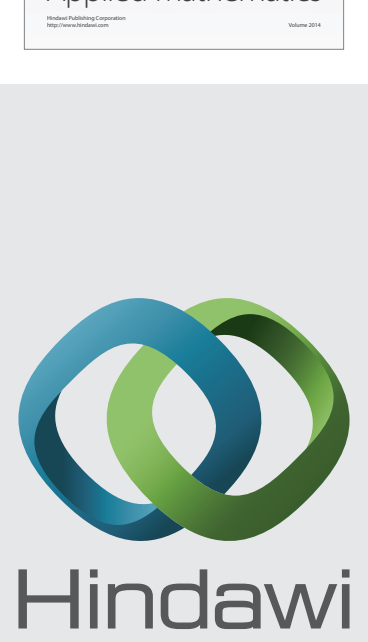

Submit your manuscripts at http://www.hindawi.com
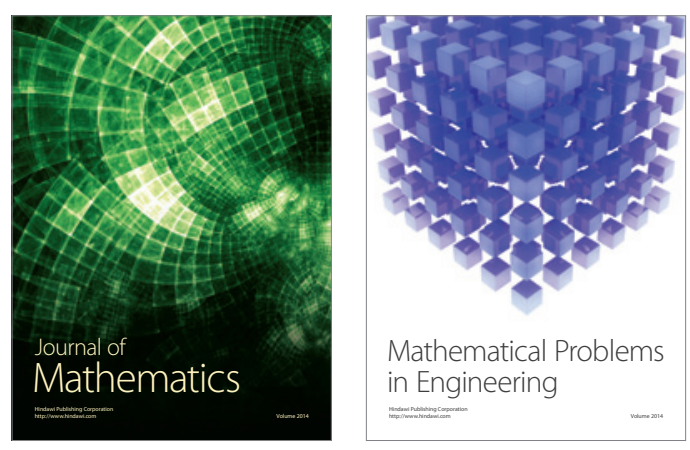

Mathematical Problems in Engineering
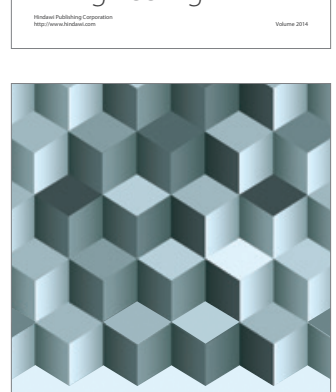

Journal of

Function Spaces
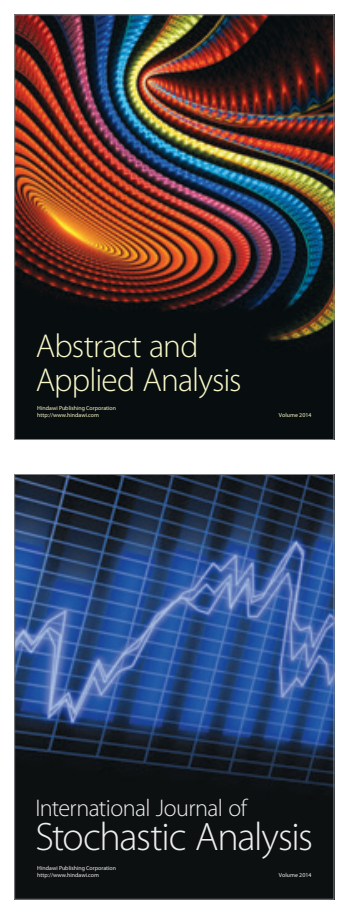

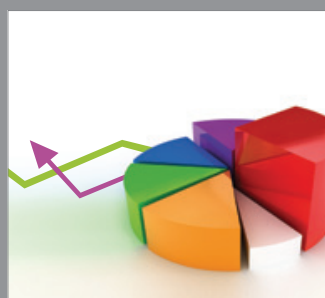

ournal of

Probability and Statistics

Promensencen
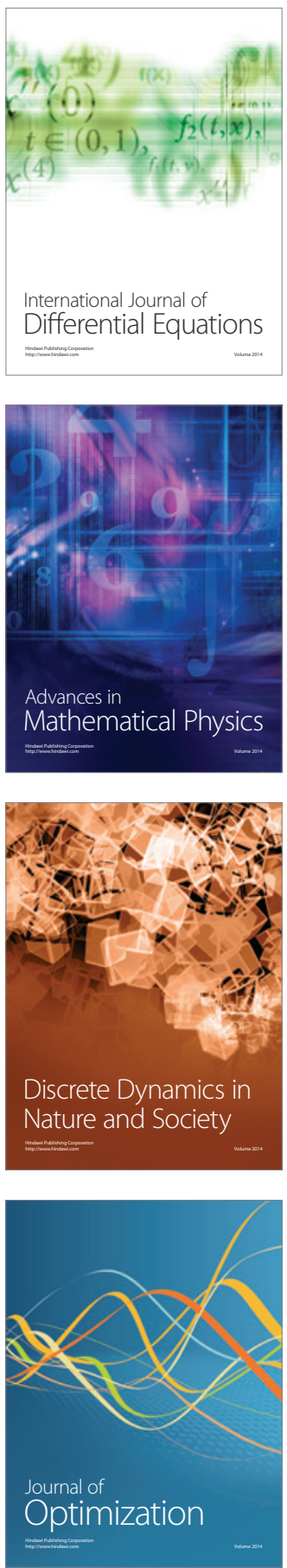